\title{
LA LABOR DEL ACADÉMICO IGNACIO TOMÁS EN ÉCIJA, SU DISPUTA CON LOS ARQUITECTOS SEVILLANOS Y LA CONSTRUCCIÓN DE LA PARROQUIA MAYOR DE SANTA CRUZ*
}

\author{
Jesús María Ruiz CARRASCO ${ }^{1}$ \\ Universidad de Córdoba
}

\begin{abstract}
Ignacio Tomás, máximo exponente de la difusión de los preceptos arquitectónicos academicistas en los reinos de Córdoba y Sevilla, intervino decisivamente en la proyección y construcción de varios edificios relevantes de Écija. Sin embargo, su labor contó con la oposición del Cabildo catedralicio, el Arzobispado y los arquitectos de Sevilla, ante quienes tuvo que defender su posición como académico. Esta disputa se manifestó singularmente en torno a la construcción de la parroquia mayor de Santa Cruz, cuyas circunstancias representan paradigmáticamente un periodo conflictivo y heterogéneo de la Arquitectura española.
\end{abstract}

Palabras clave: Academia; Ignacio Tomás; Écija; Sevilla; parroquia mayor de Santa Cruz.

\begin{abstract}
THE WORK OF THE ACADEMIC IGNACIO TOMÁS IN ÉCIJA, HIS DISPUTE WITH SEVILLIAN ARCHITECTS AND THE CONSTRUCTION OF THE PARISH CHURCH OF THE HOLY CROSS

Ignacio Tomás, maximum exponent of the diffusion of academic architectonic precepts in the kingdoms of Córdoba and Seville, intervened decisively in the projection and construction of several important buildings in Écija. Nevertheless, his work had to contend with the opposition of the cathedral chapter, the archbishopric and the architects of Seville, before whom he had to defend his position as an academic. This dispute was manifested singularly around the construction of the Holy Cross (Santa Cruz) parish church, the circumstances of which represent paradigmatically a conflicting and heterogeneous period of Spanish architecture.
\end{abstract}

Key words: Academy; Ignacio Tomás; Écija; Seville; Holy Cross parish church.

Cómo citar este artículo / Citation: Ruiz Carrasco, Jesús María (2019): "La labor del académico Ignacio Tomás en Écija, su disputa con los arquitectos sevillanos y la construcción de la parroquia mayor de Santa Cruz”. En: Archivo Español de Arte, vol. 92, núm. 365, Madrid, pp. 37-50. https://doi.org/10.3989/aearte.2019.04.

A partir de la institucionalización de la Real Academia de Bellas Artes de San Fernando en 1752, se inició un proceso divulgativo centrado en implantar una serie de valores estéticos de raigambre clásica y fundamentos ilustrados en los reinos españoles a través de la Arquitectura. Sin embargo, no fue hasta la promulgación de la Real Orden del 23 de noviembre de 1777 y,

* Este artículo forma parte de los resultados del proyecto de doctorado financiado con una ayuda FPU por el MECD (Ref.: FPU 15/02359), titulado "Cultura estética y Arquitectura de la Ilustración en Córdoba: el prelado Antonio Caballero y Góngora, el arquitecto Ignacio Tomás, la Escuela de Dibujo y la introducción de los preceptos artísticos academicistas", dirigido por Roberto González Ramos.

1192rucaj@uco.es / ORCID iD: http://orcid.org/0000-0001-6371-7437. 
especialmente, hasta la constitución de la Comisión de Arquitectura el 22 de marzo de 1786, cuando los académicos pudieron difundir sus preceptos constructivos. Como consecuencia de la fundación del organismo comisionado, la Academia realizó un notable ejercicio de evaluación de los proyectos edificativos planteados en el territorio estatal a partir de $1786^{2}$, que significó en gran medida el control formal de la Arquitectura española. No obstante, y a pesar de este avance en el intervencionismo académico, la preceptiva orden contó con la oposición de varias instituciones municipales, nobiliarias y eclesiales que promocionaron diferentes proyectos ideados por arquitectos ajenos a la Academia en determinados puntos del Reino. Asimismo, los académicos tuvieron que disputar a dichas instituciones y a sus alarifes las reformas de relevantes obras comenzadas con anterioridad a la promulgación de la resolución real, que se hallaban inconclusas a la espera de un proyecto capaz de enmendarlas. Estas circunstancias se manifestaron con especial intensidad en los reinos andaluces, donde la importancia de los gremios, el arraigo del Barroco tradicional y el rechazo al arbitraje académico, impidieron la completa implantación de los valores estéticos oficiales, al igual que generaron un conflicto que condicionó formalmente la mayoría de las edificaciones planteadas a finales del siglo XVIII. En este contexto, como paradigma de la labor de los arquitectos académicos y el conflicto originado por su presencia en dichos reinos, sobresale el caso protagonizado por Ignacio Tomás en Écija, que destacó singularmente por su intervención en la parroquia mayor de Santa Cruz.

Gracias a un extraordinario auge cultural, social y económico, Écija se erigió como uno de los centros artísticos más destacados de la geografía andaluza durante los siglos XVII y XVIII ${ }^{3}$. En concreto, fue durante la segunda mitad de la centuria dieciochista cuando, contando con casi 30.000 habitantes ${ }^{4}$, una economía próspera, seis parroquias, veinte conventos y una acaudalada nobleza ${ }^{5}$, la ciudad vivió un periodo de máximo esplendor en el que se emprendieron numerosas iniciativas artísticas. Esto fue posible también gracias a la formación y a la revitalización de talleres dedicados a oficios artesanales y artísticos en la población astigitana (sobre todo aquellos relacionados con la talla en madera ${ }^{6}$, los cuales se sumaron a la importación habitual de artífices de Sevilla y Córdoba. Así, los gremios adquirieron un papel primordial en esta ciudad, donde su labor no sólo contribuyó al progreso de la misma, sino que también posibilitó el desarrollo del decorativismo barroco que caracteriza a gran parte de sus conjuntos monumentales ${ }^{7}$. No obstante, este aumento de la promoción artística y constructiva en Écija no fue causado únicamente por su prosperidad económica, sino que también derivó de la necesidad de reedificar aquellas fábricas dañadas por el terremoto de Lisboa de 1755, que mayoritariamente correspondieron a las iglesias de la ciudad.

La primitiva parroquia mayor de Santa Cruz, de origen medieval, fue uno de los edificios más dañados por el terremoto de Lisboa. Tras el seísmo, si bien se llevaron a cabo primeramente varios reconocimientos de la fábrica parroquial a fin de repararla ${ }^{8}$, los responsables de la misma tomaron en 1763 la decisión de construir un templo de nueva planta9. Sin embargo, los

\footnotetext{
2 Dado el número de proyectos arquitectónicos evaluados por la Comisión de Arquitectura durante los citados años: novecientos setenta y tres entre 1786 y 1790 (Junta ordinaria del 3 de julio de 1791, Archivo de la Real Academia de San Fernando, Madrid [ARABASF], Secretario general, Libros de actas de juntas ordinarias, generales y públicas, legajo 3-85, f. 163r), setecientos treinta y uno desde 1790 a 1793 (Real Academia de San Fernando 1793: 12) y trescientos noventa y tres entre 1793 y 1796 (Real Academia de San Fernando 1796: 20).

3 Cabe destacar las importantes edificaciones que se erigieron en Écija durante dichas centurias. Sobre las mismas véase Morales, 2011: 33-75.

${ }^{4}$ Candau Chacón, 1995: 4.

5 Recio Mir, 2009: 381-385.

6 Sobre los tallistas ecijanos consúltese Fernández Martín, 1994.

7 En relación a los ornamentos de los edificios sevillanos en el Barroco véase Morales, 2010. Sobre la continuación de los modelos barrocos en Écija hasta el siglo XIX consúltese Fernández Martín, 1998: 591-606.

8 Sancho Corbacho, 1952: 251. Por otro lado, la torre de la parroquia mayor de Santa Cruz, atribuida a Hernán Ruiz II (Morales, 1996, p. 74), fue objeto de dos reparaciones: la primera realizada tras el terremoto de 1755 y la segunda efectuada en el año 1772 (Valseca Castillo, 1996: 41).

${ }^{9}$ Iniciativa que contó con la aprobación del Cabildo Municipal de Écija el 27 de septiembre de 1763. Hernández Díaz/ Sancho Corbacho/ Collantes de Terán, 1751: 277.
} 


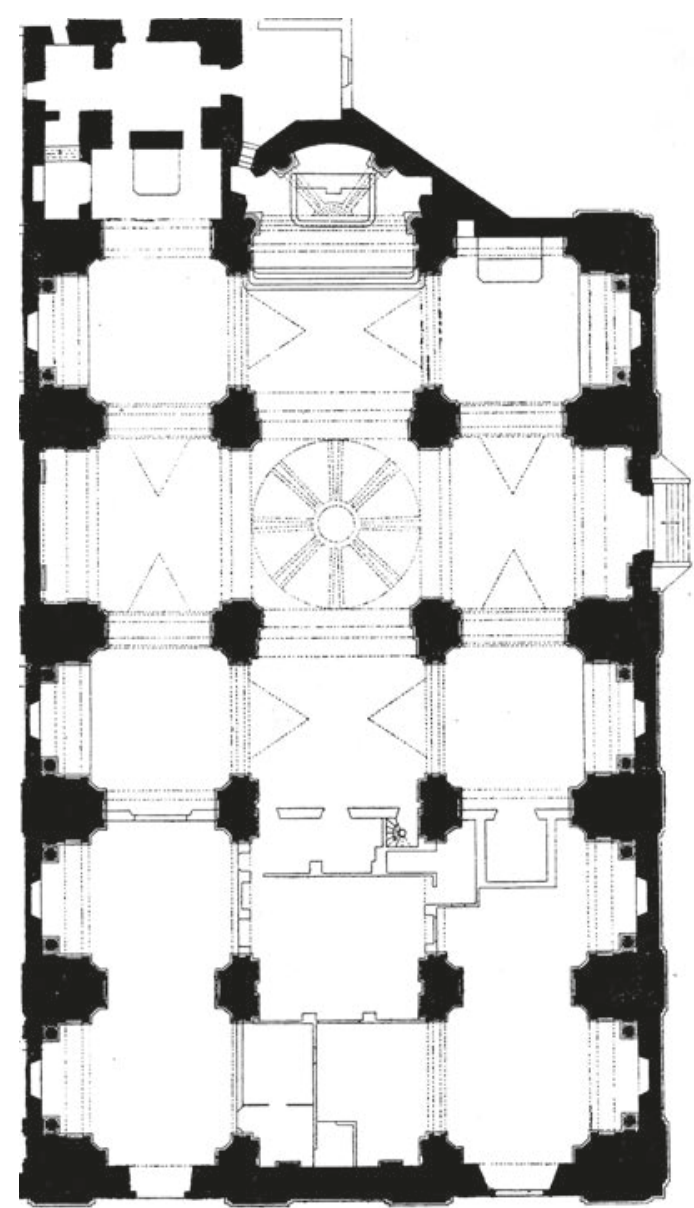

Fig. 1. Planta actual de la parroquia mayor de Santa Cruz de Écija. Fuente: Hernández Díaz/ Sancho Corbacho/ Collantes de Terán, 1751: 110. trabajos de demolición de la antigua iglesia no se iniciaron hasta el 2 de enero de 1776, y la primera piedra del nuevo edificio, diseñado por el alarife hispalense José Álvarez, no se colocó hasta el 9 de abril de $1778^{10}$. La planta propuesta por Álvarez ${ }^{11}$ se componía de un espacio de 60 varas $(50,15$ metros) de longitud y 40 varas (33,44 metros) de anchura ${ }^{12}$, ocupando una superficie total de 1.677 metros cuadrados que reflejan la magnitud del ambicioso proyecto ideado por el maestro sevillano (fig. 1). Asimismo, la iglesia debía estar distribuida en tres naves (todas ellas cubiertas con bóvedas de arista) con capillas laterales, un transepto que no sobresaliera en planta, una cúpula gallonada sobre el crucero y un ábside destacado al final de la nave principal, que estaría dividida en cinco tramos, incluidos los arcos torales.

Las obras, supervisadas por Pedro de Silva $^{13}$ desde 1778 a 1781 y por José Álvarez entre 1781 y 1785 , avanzaron con presteza a pesar de sus dimensiones; hasta tal punto que en el informe redactado por Álvarez en 1783 se notificó que el nuevo templo parroquial se encontraba levantado unas 14 varas (11,7 metros), así como ejecutados varios de los arcos formeros de las naves ${ }^{14}$. Sin embargo, el futuro edificio comenzó a ser "víctima de sus propias proporciones" $15 \mathrm{y}$, tras la designación del maestro Antonio Figueroa ${ }^{16}$ como supervisor, éste empezó a detectar las debilidades de la obra. Concretamente, tras una visita efectuada junto a Fernando Rosales ${ }^{17}$, Figueroa redactó en 1788 un informe en el que advertía la debilidad de los cimientos del edificio y la excesiva carga que soportaban sus pilares, para cuyo remedio propuso disminuir la elevación de sus bóvedas ${ }^{18}$. Igualmente, Figueroa presentó ese mismo año otro informe acompañado de una modificación en las trazas originales de José Álvarez, donde incluyó unas bóvedas subterráneas a los pies de la iglesia que cubrirían tres espacios dedicados a albergar sepulturas y que favorecerían la fortaleza de los cimientos ${ }^{19}$. Aun así, a pesar de las medidas propuestas por Figueroa para enmendar la

${ }^{10}$ Libro de defunciones de 1778 a 1795, 9 de abril de 1778, Archivo de la Parroquia de San Gil de Écija (APSGE), Defunciones, libro 5, f. 195r.

11 Contenida en Luna Fernández-Aramburu/ Serrano Barberá, 1980: 69-70.

12 Higuera Meléndez, 2016: 145-147.

13 Sobre el mismo trata Falcón Márquez, 1979.

${ }^{14}$ Écija. Santa Cruz, 5 de noviembre de 1783, Archivo General del Arzobispado de Sevilla (AGAS), Justicia, Pleitos Ordinarios, legajo 10319, exp. 1, ff. 316r-321v.

15 En palabras de Sancho Corbacho, 1952: 252.

16 Estudiado en Nogales Márquez, 2015.

17 Sobre este arquitecto véase Ollero Lobato, 2004: 383-404.

18 Écija. Santa Cruz, 21 de julio de 1788, AGAS, Justicia, Pleitos Ordinarios, legajo 10319, exp. 1, ff. 351r-357v.

19 Higuera Meléndez, 2016: 149-150. 
solidez de la parroquia mayor de Santa Cruz, la edificación de la misma se llevó a cabo sin tener en cuenta su criterio.

Tras la fundación de la Comisión de Arquitectura y como parte de la política artística emprendida por la Monarquía española, el conde de Floridablanca remitió en 1788 una comunicación al Arzobispado de Sevilla en la que se ordenaba detener todas las obras de la Archidiócesis que se encontrasen en proceso de ejecución, argumentado que estaban "dirigidas por meros maestros albañiles incapaces de ello y contra lo que tantas veces ha prevenido su majestad"20. En consecuencia, la prelatura hispalense ejecutó en diciembre del mismo año la Real Orden ${ }^{21}$, que en el caso de Écija significó la paralización de las obras parroquiales de Santa María, Santa Cruz, San Juan Bautista y Santa Bárbara ${ }^{22}$. Las particularidades constructivas de cada una de estas cuatro edificaciones inconclusas fueron atendidas por la Corona, tal y como atestigua otra misiva remitida por Floridablanca en 1789 al Arzobispado hispalense, cuyo contenido decretaba la continuación de la construcción de las iglesias de Santa María y Santa Cruz, dado su avanzado estado, así como la paralización de las obras de San Juan Bautista y Santa Bárbara hasta contar sus respectivos proyectos con la aprobación de la Real Academia de San Fernando ${ }^{23}$. Fue a partir de este momento cuando, a pesar de la pronta terminación de la ornamentada iglesia de Santa María $^{24}$, los académicos pudieron intervenir y evaluar los proyectos constructivos de Écija e iniciar así la implantación de los preceptos arquitectónicos academicistas en la ciudad. Este proceso pudo llevarse a efecto gracias a la llegada en 1790 del académico Ignacio Tomás a Córdoba, cuya proximidad a Écija propició su participación en la proyección y construcción de las parroquias de Santa Bárbara, San Juan y Santa Cruz.

Ignacio Tomás nació entre 1744 y 1746 en la ciudad catalana de Cervera ${ }^{25}$. Posteriormente, en 1767, ingresó en la Real Academia de San Fernando como alumno de la sección de Arquitectura $^{26}$, disciplina por la cual se le distinguió como "académico de mérito" en $1774^{27}$. Fue a partir de esta concesión cuando Tomás comenzó a desarrollar su actividad como académico destacado: asistiendo a Ventura Rodríguez, Antonio Plo o Francisco Sabatini ${ }^{28}$; a partir de 1780 como arquitecto del Infante Don Luis ${ }^{29}$; como miembro de la Comisión de Arquitectura en varias de sus juntas ${ }^{30}$; y como arquitecto a lo largo y ancho del Reino. En febrero de 1790 Tomás se trasladó a Córdoba ${ }^{31}$ para ocupar el puesto de director de Arquitectura de la Escuela de Dibujo ${ }^{32}$ promovida por el obispo Antonio Caballero y Góngora ${ }^{33}$. Este nombramiento, que determinó el establecimiento permanente del arquitecto catalán en Córdoba, significó también su designación como máximo responsable de las obras de la Diócesis cordobesa en $1790^{34}$ y de su Catedral en $1791^{35}$.

20 Fernández Martín, 2000: 67.

${ }^{21}$ Ecija. San Juan, 1 de diciembre de 1788, AGAS, Justicia, Pleitos Ordinarios, legajo 11580, exp. 6, s/f.

22 Martín Ojeda/ García León, 1995: 162.

23 Écija. Santa Cruz, 2 de marzo de 1789, AGAS, Justicia, Pleitos Ordinarios, legajo 10319, exp. 1, ff. 370r-370v.

${ }^{24}$ Écija. Santa María, 30 de abril de 1795, AGAS, Justicia, Pleitos Ordinarios, legajo 10691, exp. 4, ff. 568r-569r.

25 Diferentes datos sobre el nacimiento y la ascendencia de Ignacio Tomás aparecen contenidos en Valverde Madrid: 296-300. Serra Masdeu, 2014: 129-141. Ruiz Carrasco, 2017: 343-370.

${ }^{26}$ Libro en donde se sientan los discípulos de esta Real Academia de San Fernando, desde el año 1752 en adelante, 1752-1778, ARABASF, Secretario general, Libros de Matricula, legajo 3-300, f. 57r.

27 García Sepúlveda/ Navarrete Martínez, 2007: 415.

28 Sambricio, 1986: 423.

29 Sambricio, 1985: 262.

30 Juntas de la Comisión de Arquitectura n 7, 12 y 61, 10 de agosto de 1786, 9 de noviembre de 1786 y 15 de enero de 1790, ARABASF, Secretario general, Libros de Actas de la Comisión de Arquitectura, legajo 3-139, ff. 40r, 45r. y 134 r.

31 Junta de la Comisión de Arquitectura $n^{\circ}$ 62, 5 de febrero de 1790, ARABASF, Secretario general, Libros de Actas de la Comisión de Arquitectura, legajo 3-139, f. 136r.

32 La Escuela de Dibujo de Córdoba ha sido tratada en Aranda Doncel, 1986: 33-49.

33 Sobre el mismo véase Pérez Ayala, 1951.

34 Rivas Carmona, 1986: 45-48.

35 Concretamente, el 24 de marzo de dicho año. Tercer Libro de Cuentas dadas por Juan de Castro. Desde 1787 a 1791, 3 de agosto de 1791, Archivo de la Catedral de Córdoba (ACC), Cuentas de Fábrica, legajo 4042, s/f. 
Teniendo en cuenta estas circunstancias y las antes reseñadas, la Comisión de Arquitectura sugirió en marzo de 1790 a Tomás como delineante de la futura iglesia parroquial de Santa Bárbara, tras la desestimación de un proyecto planteado por Antonio Figueroa para su construcción ${ }^{36}$. Una vez conformados los planos ${ }^{37}$ y redactado el correspondiente informe por parte de Ignacio Tomás en octubre de $1790^{38}$, los comisionados aprobaron el proyecto que éste realizó para dicha iglesia en diciembre de 1790, cuando además se propuso al arquitecto catalán como director de la obra ${ }^{39}$. A partir de entonces Tomás se erigió como referente arquitectónico en Écija, labor que compaginó con su actividad en Córdoba y que implicó su participación en las obras más importantes de la población ecijana durante la última década del siglo XVIII, especialmente en la reforma constructiva de la parroquia mayor de Santa Cruz.

En las mismas fechas de la asignación de Tomás como delineante y director de la obra parroquial de Santa Bárbara, la edificación de Santa Cruz se encontraba en 1790 bajo la supervisión del arquitecto sevillano Antonio Figueroa, gracias a que el conde de Floridablanca permitió continuar la construcción del edificio sin la supervisión de la Real Academia de San Fernando. Figueroa, aún habiendo reducido 4,25 varas (3,55 metros) la altura de las bóvedas de la iglesia, continuó mostrando dudas sobre la solidez del edificio tras supervisar la obra en junio de $1790^{40}$. Estas inseguridades propiciaron que la Archidiócesis dispusiera la visita de los alarifes ecijanos Francisco Aguilar y Simón Salazar ${ }^{41}$, quienes en un informe fechado en febrero de 1791 no consideraron ninguna modificación del proyecto original ${ }^{42}$. También fue solicitada la participación del arquitecto hispalense José Camino ${ }^{43}$, quien propuso revestir los pilares del templo con cantería en marzo del mismo año ${ }^{44}$. Por último, Figueroa elaboró paralelamente un informe mediante el cual pretendía concluir definitivamente con los problemas de solidez de la obra, añadiéndole dos suplementos a los pilares de la nave principal ${ }^{45}$. El escrito no contentó a los responsables del Arzobispado de Sevilla, quienes solicitaron el 27 de marzo al académico Ignacio Tomás que examinara la fábrica parroquial y diera su parecer sobe lo expuesto por José Camino y Antonio Figueroa ${ }^{46}$. Esta demanda significó el inicio de la participación de Tomás en la obra de la parroquia mayor de Santa Cruz, que en esta ocasión no derivó de la mediación de la Real Academia de San Fernando, sino de la confianza que la Archidiócesis hispalense depositó en el arquitecto académico a raíz de su intervención en la iglesia de Santa Bárbara de la misma ciudad.

El estado de la iglesia de Santa Cruz era tal que, en palabras de Antonio Ponz, se vendría "abajo antes de concluirla" si no se enmendaban "errores muy clásicos que todos advierten, no solo en la decoración, sino" también "en la solidez" 47 . Errores que Ignacio Tomás tendría la ocasión de afrontar. Como consecuencia de la demanda de la Archidiócesis hispalense, el arquitecto académico redactó un informe en mayo de 1791, donde señaló que Antonio Figueroa "no dio con la principal enfermedad" del edificio pero sí "acertó con la medicina" que se debía aplicar al mismo. Si bien estimaba que el aumento del grueso de los pilares consolidaría la iglesia, entendía que sólo los zócalos de los suplementos destinados a reforzar los pilares debían estar

36 Junta de la Comisión de Arquitectura $n^{\circ}$ 64, 30 de marzo de 1790, ARABASF, Secretario general, Libros de Actas de la Comisión de Arquitectura, legajo 3-139, f. 139v.

37 García León, 1990: 169-188.

${ }^{38}$ El informe constructivo aparece contenido en Écija. Santa Bárbara, 28 de octubre de 1790, AGAS, Justicia, Pleitos Ordinarios, legajo 10319, exp. 2, ff. 116r-121v.

39 Junta de la Comisión de Arquitectura $n^{\circ} 71,1$ de diciembre de 1790, ARABASF, Secretario general, Libros de Actas de la Comisión de Arquitectura, legajo 3-139, f. 152v.

${ }^{40}$ Écija. Santa Cruz, junio de 1790, AGAS, Justicia, Pleitos Ordinarios, legajo 10319, exp. 1, ff. 378r-381v.

41 Siendo Francisco de Aguilar maestro mayor de obras de la ciudad ecijana y Simón Salazar su ayudante. Martín Pradas, 1995: 68-69.

42 Écija. Santa Cruz, 12 de febrero de 1791, AGAS, Justicia, Pleitos Ordinarios, legajo 10319, exp. 1, ff. 385r-386r.

43 Sobre José Camino véase Ollero Lobato, 1994: 85-86.

44 Écija. Santa Cruz, 2 de marzo de 1791, AGAS, Justicia, Pleitos Ordinarios, legajo 10319, exp. 1, ff. 387r-390r.

45 Écija. Santa Cruz, 21 de marzo de 1791, AGAS, Justicia, Pleitos Ordinarios, legajo 10319, exp. 1, ff. 391r-395r.

46 Écija. Santa Cruz, 2 de mayo de 1791, AGAS, Justicia, Pleitos Ordinarios, legajo 10319, exp. 1, f. 397r.

47 Ponz, 1792: 161. 


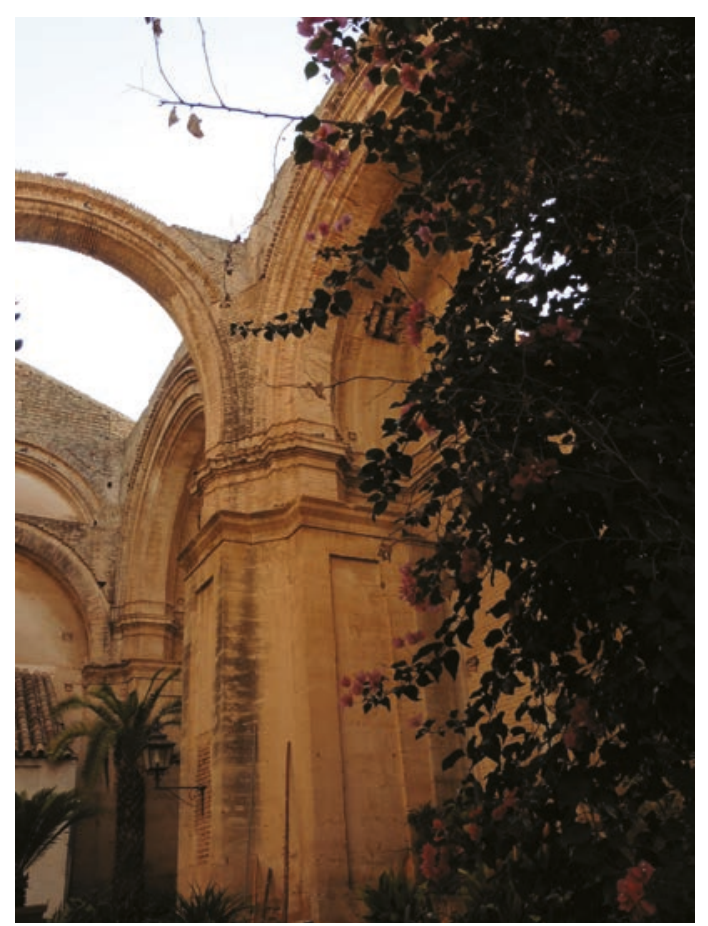

Fig. 2. Vista de los pilares originales de la iglesia de Santa Cruz y de los suplementos ideados por Ignacio Tomás para la misma. Fotografía del autor.

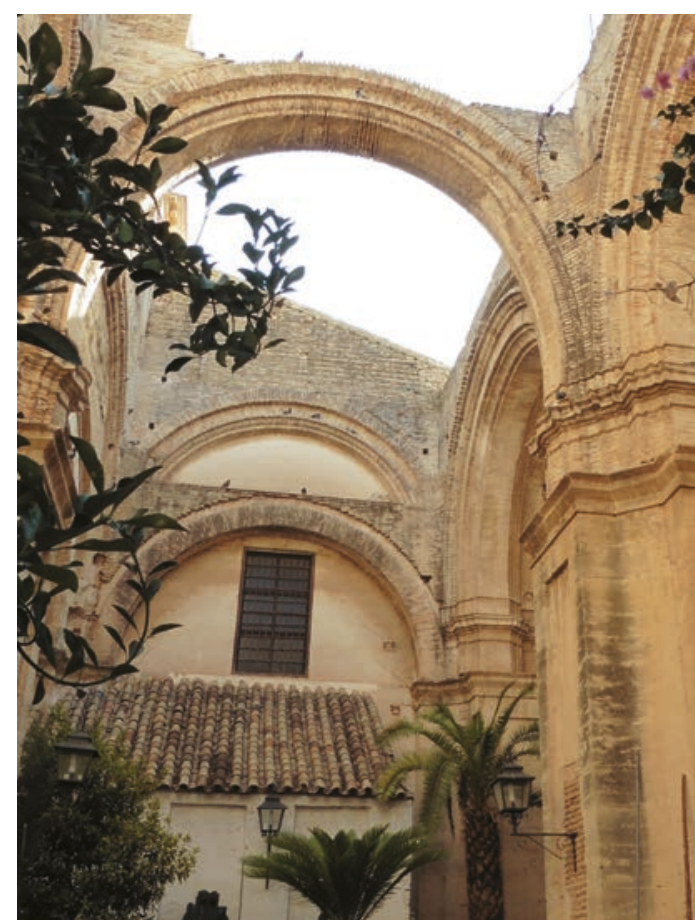

Fig. 4. Diferencia de altura entre los arcos de las naves laterales del proyecto original y los dispuestos por Tomás. Fotografía del autor.

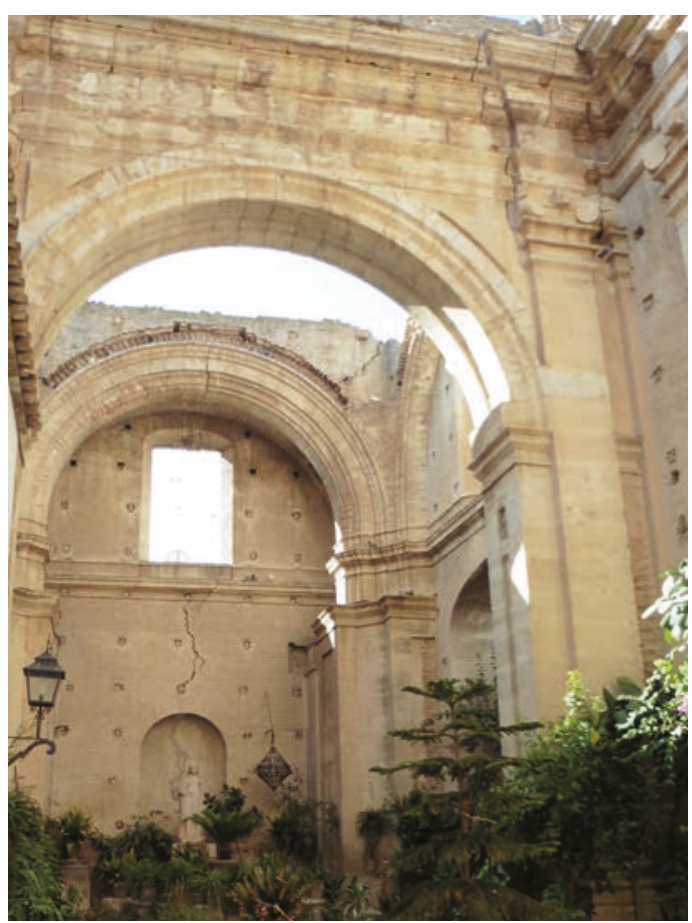

Fig. 3. Tramos inacabados de la iglesia donde se aprecia la reducción de los arcos propuesta por Tomás. Fotografía del autor.

realizados de cantería, siendo los restantes de ladrillo y mezcla (la mitad de argamasa y la otra mitad de yeso), uniendo así dichos suplementos con los pilares ya construidos, contribuyendo a la estabilidad estructural del edificio y abaratando costes ${ }^{48}$. La inclusión de los citados suplementos conllevaría la disminución de la altura y de la luz de los arcos de las naves de la iglesia, ya que el ensanchamiento de los pilares implicaría el mismo efecto en dichos arcos, que aumentarían así su capacidad para soportar y distribuir el empuje de las bóvedas (fig. 2). Por otro lado, el buen funcionamiento de la combinación entre el ladrillo y la citada mezcla en los añadidos de los pilares, según redactó el arquitecto catalán en su informe, quedaba acreditada por su utilización en la construcción de San Francisco "el Grande" de Madrid por parte de Antonio $\mathrm{Plo}^{49}$; quien, ante

48 Écija. Santa Cruz, 2 de mayo de 1791, AGAS, Justicia, Pleitos Ordinarios, legajo 10319, exp. 1, ff. 398v-399r.

49 Con quien trabajó Ignacio Tomás en la construcción de San Francisco "el Grande" de Madrid. Sambricio, 1986: 423. 
problemas estructurales parecidos a los de la iglesia de Santa Cruz de Écija, empleó dicha técnica aconsejado por el propio Tomás ${ }^{50}$.

Para complementar la solidez que debían aportar los suplementos de los pilares a la iglesia de Santa Cruz, Tomás aconsejó una disminución notable de la altura de sus bóvedas y de los arcos que debían sostenerlas respecto al proyecto original y a las posteriores reformas del edificio (fig. 3). Tomando como referencia la anchura de la nave principal, de 31 pies (8,64 metros), y en base a "la proporción entre sesquiáltera y dupla" ${ }^{1}$, Tomás calculó que la altura de la nave principal y de los arcos torales hasta su correspondiente clave debía de ser de 54 pies (15,05 metros). Asimismo, consideraba que los 51,75 pies (14,42 metros) que alcanzaba la obra ya realizada de la iglesia de Santa Cruz hasta el arranque de la bóveda de la nave principal eran desmedidos (fig. 4), dado que si a dicha altura se le añadía la mitad de la anchura de la nave (que correspondería con la altura del semicírculo del arco toral), sumaría un total de 67,25 pies (18,74 metros) hasta la clave de la bóveda de cañón de la nave principal, excediendo así 13,75 pies (3,83 metros) lo convenido por la regla de proporcionalidad establecida por Tomás ${ }^{52}$. Gracias a sus medidas, que consistirían en la ya referida incorporación de los suplementos de los pilares, el arquitecto catalán pretendía aliviar el peso que debían soportar los cimientos y los pilares, así como dar debida proporción a todas las partes del edificio y favorecer a la solidez del mismo. De igual modo, con estas medidas aspiraba a distribuir eficazmente el peso de las bóvedas entre los diferentes elementos sustentantes, evitando así que el empuje de las fuerzas ejercidas por las bóvedas confluyera a través de las danzas de arcos formeros en la fachada (fig. 5), cuyo grueso era únicamente la tercera parte del diámetro de dichos $\operatorname{arcos}^{53}$. Todo ello perseguía solucionar los problemas estructurales que sufría la iglesia como consecuencia de la inadecuada proporción que guardaba la altura de sus bóvedas con respecto a la altura y anchura de sus pilares, hasta la redacción del informe de Tomás ${ }^{54}$.

Seguidamente, el arquitecto académico señaló en su informe que las medidas reseñadas en el mismo abaratarían considerablemente los costes de la construcción de la iglesia hasta los 30.000 reales de vellón, que supondrían menos de la cuarta parte del presupuesto planteado por Antonio Figueroa para la reforma del edificio ${ }^{55}$. Esta disminución del gasto de la obra también vendría dada por la eliminación de los "miembros y resaltes" que hubieran decorado la iglesia siguiendo el proyecto original y que Tomás consideraba "ridículos" 56 por su falta de decoro y por su alto $\operatorname{coste}^{57}$. De este modo el arquitecto académico estimaba que, en conclusión, para que el edificio adquiriera la "naturalidad, simplicidad, proporción, simetría y decoro" 58 pertinentes, se debía disminuir su altura inicial, ejecutar los refuerzos propuestos anteriormente, eliminar la decoración superflua, consolidar su estructura y modificar sus partes ya construidas. Por todo ello, Tomás recomendó a los responsables de la Archidiócesis de Sevilla que promovieran la elaboración de nuevos diseños para la edificación de la parroquia mayor de Santa Cruz de Écija, a la vez que lamentó la cantidad de fondos invertidos inútilmente hasta la fecha en "una obra de tanta consideración" 59 .

50 Écija. Santa Cruz, 2 de mayo de 1791, AGAS, Justicia, Pleitos Ordinarios, legajo 10319, exp. 1, f. 399v.

51 Que Fray Lorenzo de San Nicolas denominó como "superpartiensquarta" y que entendió como necesaria para la configuración de los alzados de los edificios. San Nicolás, 1736: 44-45.

${ }_{52}$ Écija. Santa Cruz, 2 de mayo de 1791, AGAS, Justicia, Pleitos Ordinarios, legajo 10319, exp. 1, ff. 400v-401r.

53 Sobre el adecuado grosor de las fachadas de los edificios, que no sólo deben de obedecer a la proporción con respecto a su elevación, sino también a la carga que deben soportar, véase Bails, 1783: 223-225.

${ }^{54}$ Écija. Santa Cruz, 2 de mayo de 1791, AGAS, Justicia, Pleitos Ordinarios, legajo 10319, exp. 1, ff. 397r-398r.

55 Écija. Santa Cruz, 2 de mayo de 1791, AGAS, Justicia, Pleitos Ordinarios, legajo 10319, exp. 1, f. 400r.

${ }^{56}$ El propio Tomás señaló en su informe constructivo para la iglesia de Santa Bárbara de Écija la "barbarie y mal gusto" de los retablos y los elementos decorativos incorporados a las edificaciones ecijanas barrocas. Écija. Santa Bárbara, 28 de octubre de 1790, AGAS, Justicia, Pleitos Ordinarios, legajo 10319, exp. 2, f. 118r.

57 Écija. Santa Cruz, 2 de mayo de 1791, AGAS, Justicia, Pleitos Ordinarios, legajo 10319, exp. 1, f. 401r.

58 Virtudes esenciales que según los académicos debía tener cualquier proyecto constructivo. Dicha terminología aparece expuesta en García Melero, 1995: 271.

59 Écija. Santa Cruz, 2 de mayo de 1791, AGAS, Justicia, Pleitos Ordinarios, legajo 10319, exp. 1, ff. 401r-402r. 


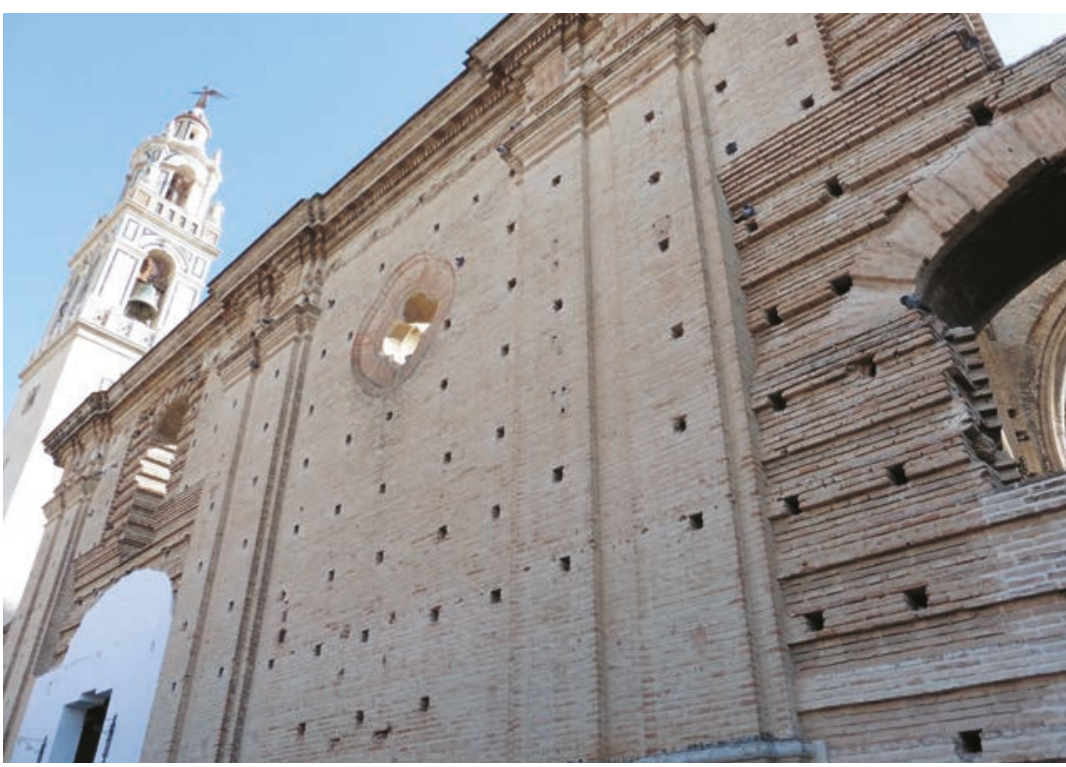

Fig. 5. Fachada inconclusa de la iglesia. Fotografía del autor.

Como consecuencia del referido informe, la Archidiócesis de Sevilla le encargó el 24 de mayo de 1791 a Ignacio Tomás la elaboración de un nuevo proyecto para la edificación de la parroquia mayor de Santa Cruz de Écija, el cual no concluyó hasta el 14 de noviembre del mismo año ${ }^{60}$. Este lapso de tiempo entre el encargo del nuevo plan y su finalización se explica porque, entre otras ocupaciones, el arquitecto catalán tuvo que afrontar desde el 8 de abril la compleja planificación de la parroquia San Juan de Écija, cuyos diseños definitivos no fueron terminados hasta el 25 de febrero de 1792 y aprobados por la Real Academia de San Fernando hasta el 21 de abril del mismo año ${ }^{61}$. Con respecto al proyecto de la parroquia mayor de Santa Cruz, Tomás presentó cuatro diseños que no se han conservado y un informe constructivo que los acompañaba, el cual continuaba las directrices aportadas en el escrito anteriormente desarrollado. Sin embargo, el arquitecto académico rectificó la altura de la bóveda principal, calculando que ésta debía elevarse algo más de los 54 pies $(15,05$ metros $)$ propuestos en su primer informe, a fin de otorgar "alguna regular elevación a los arcos y bóvedas de las naves chicas", pero disminuyendo en 16 pies (4,45 metros) la altura del proyecto inicial de la obra ${ }^{62}$. Tomás también corrigió la composición de los suplementos de los pilares, que debían estar compuestos de ladrillos provenientes de los arcos de la fábrica original que iban a ser reemplazados, y compactados por una mezcla compuesta por cuatro partes de argamasa y una de yeso ${ }^{63}$; así como elevados hasta una altura no superior a los 24 pies (6,69 metros). Del mismo material que los citados suplementos, se llevarían a cabo los arcos torales y formeros, las pechinas, la cúpula principal, su linterna, la bóveda de la nave principal y las bóvedas baídas de las laterales, las cuales estarían abiertas en su clave por un anillo circular que abriría una claraboya para favorecer la entrada de luz. Asimismo, Tomás planificó la ejecución de un frontón triangular sobre el cuerpo dórico de la fachada principal (el

${ }^{60}$ Écija. Santa Cruz, 14 de noviembre de 1791, AGAS, Justicia, Pleitos Ordinarios, legajo 10319, exp. 1, ff. 405r$407 \mathrm{v}$.

61 Proyecto que Tomás afrontó a petición de los responsables de la fábrica parroquial y bajo la supervisión de la Real Academia de San Fernando. Véanse las resoluciones de la Comisión de Arquitectura sobre la adjudicación y aprobación definitiva de la obra en Junta de la Comisión de Arquitectura $n^{\circ} 76$ y 87, 8 de abril de 1791 y 21 de abril de 1792, ARABASF, Secretario general, Libros de Actas de la Comisión de Arquitectura, legajo 3-139, ff. 159r, 187r-187v.

62 Écija. Santa Cruz, 14 de noviembre de 1791, AGAS, Justicia, Pleitos Ordinarios, legajo 10319, exp. 1, ff. 405r$405 \mathrm{v}$.

${ }^{63}$ Composición que ya propuso Ignacio Tomás para los arcos de la iglesia de Santa Bárbara. Écija. Santa Bárbara, 28 de octubre de 1790, AGAS, Justicia, Pleitos Ordinarios, legajo 10319, exp. 2, f. 119v. 


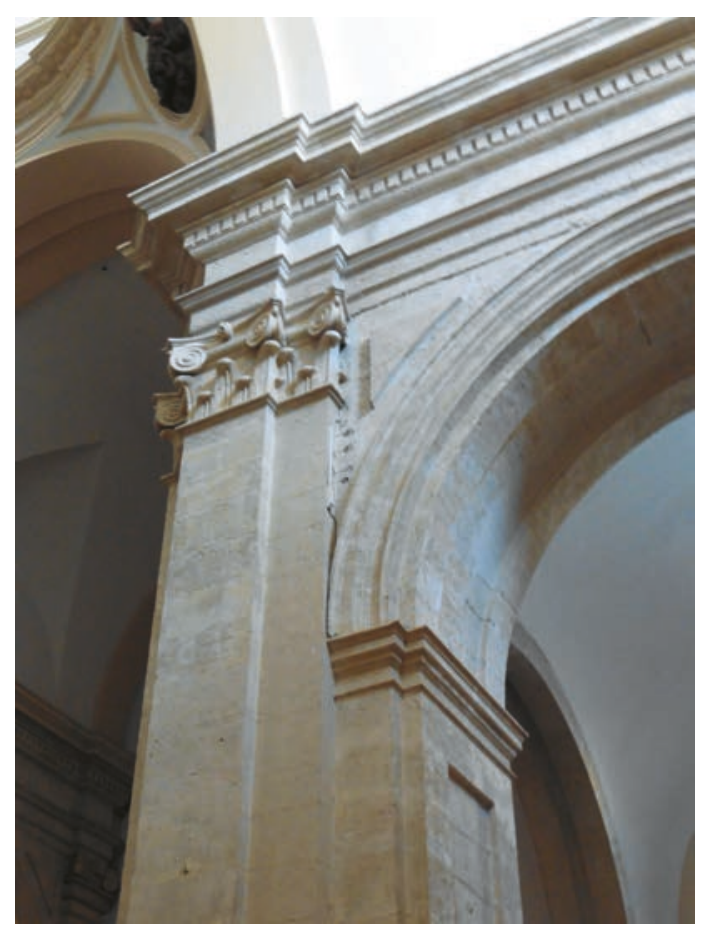

Fig. 6. Pilares de piedra del interior de la iglesia. Fotografía del autor.

cual correspondería con la caída de las cubiertas) al igual que una cornisa que recorrería el perfil de la nave principal, del transepto y del presbiterio, que serviría además de alero para los tejados ${ }^{64}$. Por último, el arquitecto escogió un modelo de cubiertas de "contrabóvedas escarzanas de ladrillo tabicado"65, tanto por su seguridad, como por su capacidad para repartir mejor los pesos en una obra de tales dimensiones ${ }^{66}$. Todo ello completó y especificó las directrices aportadas por Ignacio Tomás en su primer informe para la correcta construcción de la parroquia mayor de Santa Cruz y su adaptación a los preceptos arquitectónicos academicistas.

Con el propósito de comprobar la conveniencia de lo propuesto por Tomás, el Arzobispado y el Cabildo de la Catedral de Sevilla convocaron en marzo de 1792 al maestro mayor de obras de la institución catedralicia, Manuel Núñez ${ }^{67}$, para que visitara la obra de Santa Cruz y analizara los informes elaborados hasta la fecha sobre la misma ${ }^{68}$. Nuñez, si bien alabó la relevancia teórica y práctica de Ignacio Tomás como arquitecto, así como sus diseños para la iglesia que nos ocupa, criticó el compuesto ideado por el académico para los suplementos de los pilares de la obra, dado que entendía que el yeso y la argamasa eran incompatibles. En este sentido, informó que los suplementos ya elaborados bajo la dirección de Tomás se hallaban defectuosos y despegados de la fábrica original, lo cual confirmaba la incompatibilidad entre el yeso y la argamasa advertida por Nuñez, al igual que el error consciente del académico al planificar dicha mezcla ${ }^{69}$. Por ello, y advirtiendo que las bóvedas debían estar compuestas por una conjunción de yeso y arena o por "yeso puro", Núñez defendió que los suplementos de los pilares de la obra de Santa Cruz debían ser de piedra, dada su perdurabilidad y su capacidad para asegurar la fábrica antigua (fig. 6); a la que deberían estar unidos por medio de hiladas alternas de cola de milano y mezcla de cal y arena ${ }^{70}$. Por otro lado, en un apéndice del informe de Núñez añadido el 20 de abril, éste advirtió que la nave mayor debería estar sujeta mediante una serie de vigas de madera de Flandes dispuestas sobre las naves laterales para reforzar la sujeción del conjunto, eliminando así las linternas planteadas por Tomás para las mismas ${ }^{71}$ (fig. 7).

${ }^{64}$ Écija. Santa Cruz, 14 de noviembre de 1791, AGAS, Justicia, Pleitos Ordinarios, legajo 10319, exp. 1, ff. 405v-406v.

65 También se ha alabado desde el ámbito académico las virtudes de este tipo de bóvedas por su capacidad para evitar la propagación de incendios. Bails, 1783: 374.

${ }^{66}$ Écija. Santa Cruz, 14 de noviembre de 1791, AGAS, Justicia, Pleitos Ordinarios, legajo 10319, exp. 1, ff. 407r$407 \mathrm{v}$. En contraposición, para la construcción de la iglesia de Santa Bárbara eligió un tipo de cubiertas de madera que aparece reseñado en Écija. Santa Bárbara, 28 de octubre de 1790, AGAS, Justicia, Pleitos Ordinarios, legajo 10319, exp. 2, f. 119v.

${ }_{67}$ Quien ostentó este cargo desde 1770 hasta 1802. Sobre este arquitecto véase Ollero Lobato, 2004: 423-433.

68 Écija. Santa Cruz, 20 de marzo de 1792, AGAS, Justicia, Pleitos Ordinarios, legajo 10319, exp. 1, ff. 409r-415v.

${ }^{69}$ Cabe destacar que Nuñez rechazó cuatro años más tarde el proyecto del académico Julián de Barcenilla para la iglesia de San Ildefonso de Sevilla. Quiles García, 1997: 317-319.

70 Écija. Santa Cruz, 20 de marzo de 1792, AGAS, Justicia, Pleitos Ordinarios, legajo 10319, exp. 1, ff. 410r-415v.

71 Écija. Santa Cruz, 20 de abril de 1792, AGAS, Justicia, Pleitos Ordinarios, legajo 10319, exp. 1, ff. 415v-416v. 
Tras la recepción del informe redactado por Manuel Núñez, el provisor del Cabildo de la Catedral hispalense, Miguel Rul, autorizó el 9 de mayo el comienzo de las obras de la parroquia mayor de Santa Cruz de Écija siguiendo los diseños de Ignacio Tomás y el dictamen de Núñez ${ }^{72}$. Esta última circunstancia provocó la indignación del arquitecto académico, quien, en un escrito fechado el 9 de julio, defendió su posición ante el citado Miguel Rul, denunciando la conducta del director de la obra ${ }^{73}$, José Jiménez Henestrosa ${ }^{74}$. Advirtiendo que la actuación de Henestrosa resultaba perjudicial para los intereses de los partícipes en diezmos y la solidez del edificio, Tomás le acusó de propiciar que la obra fuera supervisada extraoficialmente por Antonio Figueroa, cuya presencia relacionaba directamente con la deficiencia percibida en los suplementos de los pilares que el académico había ideado para consolidar la iglesia. Igualmente, Tomás mostró su indignación ante la visita de Manuel Nuñez, la cual entendía que incumplía el capítulo 34 de los Estatutos de la Real Academia de Bellas Artes de San Fernando, que establecía que "ningún juez o tribunal" podía obligar a los académicos a "ser visitados de veedores o síndicos" 75 . Por último, Tomás advirtió que si no cesaba dicha situación estaría obligado a denunciarla ante la Corona por medio de la Real Academia de San Fernando, a fin de que se tomaran medidas al respecto ${ }^{76}$.

Dado que la denuncia de Tomás fue ignorada y las obras comenzaron a ejecutarse el 23 de julio según lo dictado por Miguel Rul, el académico redactó el 22 de agosto una demanda (acompañada de otro escrito aclaratorio) enviada tres días más tarde a la Real Academia de San Fernando, mediante la cual solicitaba la intermediación del Rey para que el Cabildo de la Catedral y el Arzobispado de Sevilla le proporcionaran los expedientes en los que constaba la "ofensa" que los alarifes hispalenses emitieron contra él por su participación en la construcción de la iglesia de Santa Cruz de Écija, a fin de poder probar su situación ante un tribunal ${ }^{77}$. Para argumentar la importancia de su solicitud, Tomás expuso detalladamente sus circunstancias. Destacó la oposición que los académicos "salidos de la corte" padecían particularmente en Andalucía, donde se veían desamparados y sin apoyo para ejercer su ocupación, frente a los "maestros albañiles" tradicionales ${ }^{78}$, quienes, "por varias ruinas e intereses", contaban con protectores que los promocionaban y atendían a su criterio ${ }^{79}$. En este sentido, desarrolló su caso concreto asegurando que, si bien durante el año anterior de 1791 los tres diputados canónigos de la Catedral hispalense (que cambiaban anualmente) habían confiado en su labor, los nombrados al siguiente año despreciaron su trabajo hasta el punto de sólo abonarle cuatro ducados al día por cada una de las visitas efectuadas a las diferentes obras de Écija. Tomás entendía que dichos pagos, inferiores a la mitad de los que recibían los alarifes hispalenses, estaban estipulados de ese modo para que acabara renunciando a su trabajo en Écija, pues eran insuficientes para costear sus viajes desde Córdoba. Para reforzar su argumentación, contrapuso su situación a la de los arquitectos académicos en Granada (entre los que se encontraba su hermano Domingo Tomás ${ }^{80}$ ), que recibían

72 Écija. Santa Cruz, 19 de febrero de 1793, AGAS, Justicia, Pleitos Ordinarios, legajo 10319, exp. 1, ff. 425r-426r.

73 Véanse algunos ejemplos en los que la participación de diferentes arquitectos académicos en Sevilla se vio truncada por la intromisión de los comitentes de la obra (Ros González, 2000: 153-173. Fernández González, 2007: 203-228), de diferentes arquitectos sevillanos ajenos a la Academia (Ruiz Carrasco, 2018: 298-302) y del Cabildo de la Catedral de Sevilla (Baena Gallé/ Hernández Núñez, 1998: 607-623. Recio Mir, 1998: 379-398).

${ }^{74}$ Écija. Santa Cruz, 9 de julio de 1792, AGAS, Justicia, Pleitos Ordinarios, legajo 10319, exp. 1, ff. 423r-424r.

75 Écija. Santa Cruz, 9 de julio de 1792, AGAS, Justicia, Pleitos Ordinarios, legajo 10319, exp. 1, f. 423v. Destacando que lo expuesto por Tomás corresponde con el contenido de los Estatutos de la Real Academia, cabe reseñar que el capítulo 34 (el último) de los mismos es el dedicado a los privilegios de los académicos. Real Academia de San Fernando, 1757: 94-102.

${ }^{76}$ Écija. Santa Cruz, 9 de julio de 1792, AGAS, Justicia, Pleitos Ordinarios, legajo 10319, exp. 1, ff. 423v-424r.

77 Al Rey. Representacion por D. Ignacio Thomas Arquitecto y Academico de Merito de la Real Academia de San Fernando, 22 de agosto de 1792, ARABASF, Comisión de Arquitectura, Informes, Competencias profesionales, legajo 2-26-1, exp. 2, ff 1r-6r.

78 A esta cuestión se refiere a nivel estatal García Melero, 1991: 283-348.

79 Écija. Parroquia de Santa Bárbara y San Juan, 25 de agosto de 1792, ARABASF, Comisión de Arquitectura, Informes, Iglesias parroquiales, legajo 2-33-2, exp. 1, s/f.

${ }^{80}$ Sobre el mismo véase Guillén Marcos, 1990: 110-111. Sambricio, 1986: 421-423. 
Fig. 7. Sección frontal de la parroquia mayor de Santa

Cruz. Fuente: Hernández

Díaz/ Sancho Corbacho/ Collantes de Terán, 1751: 113
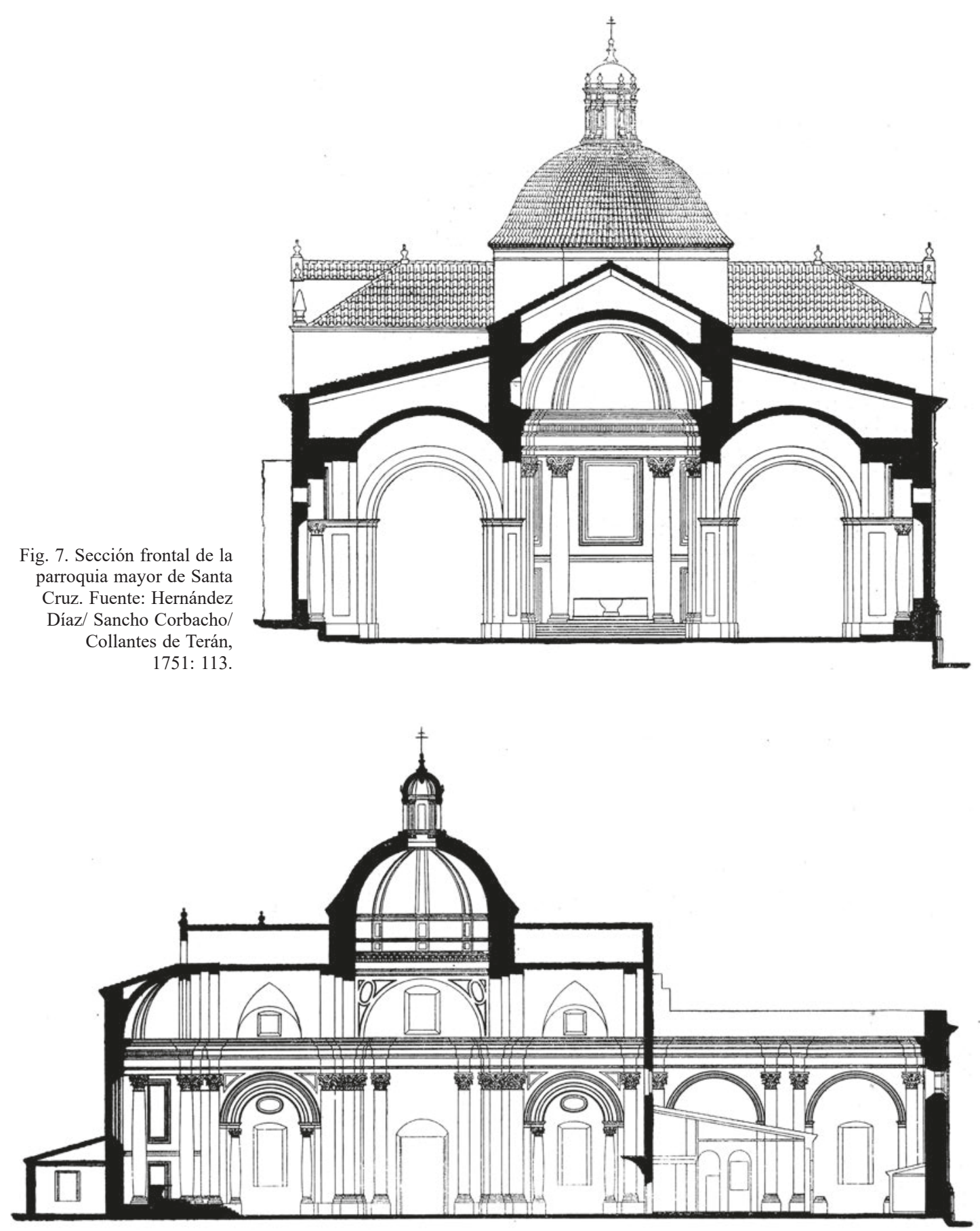

Fig. 8. Sección longitudinal de la parroquia mayor de Santa Cruz. Fuente: Hernández Díaz/ Sancho Corbacho/ Collantes de Terán, 1751: 111. 
entre 500 y 1000 ducados anuales por la dirección de las obras, así como entre 20 y 30 ducados diarios por cada una de las visitas ${ }^{81}$. Finalmente, el arquitecto académico expuso que si al Cabildo catedralicio hispalense no se le ponía "algún freno" perdería la dirección de las obras de Écija, peligraría el estado de éstas y se malgastarían excesivos caudales en las mismas; lo cual entendía que debía ser comunicado al Duque de Medinaceli, principal partícipe de los diezmos de la ciudad ecijana, para que tomara parte en el asunto ${ }^{82}$.

La demanda de Tomás fue atendida por la Junta particular de la Real Academia de San Fernando en la sesión celebrada el 2 de septiembre, donde se aprobó remitir dicha súplica al secretario de Estado (y, por tanto, protector de la Academia), el conde de Aranda, para que diera cuenta de ella ante el Rey ${ }^{83}$. Por consiguiente, Isidoro Bosarte, secretario de la Academia, envió una representación al Conde donde le comunicaba el asunto y destacaba la importancia de los sucesos y de las "obras costosas" a las que afectaban ${ }^{84}$. A pesar de todo, la Corona no intercedió en el conflicto, lo cual contrasta con el control ejercido por el conde de Floridablanca sobre las obras sacras de Écija en 1788 y 1789 que había posibilitado la intervención académica en las mismas.

Las obras de la parroquia mayor de Santa Cruz se desarrollaron intermitentemente hasta su definitiva apertura al culto el 21 de diciembre de $1836^{85}$. Su proceso constructivo dio como resultado un edificio inacabado que, a pesar de una iniciativa promovida a mediados del siglo XX para su conclusión ${ }^{86}$, presenta los dos primeros tramos de sus tres naves sin abovedar y la fachada sin rematar. Sin embargo, las partes inconclusas del edificio, además de la fachada, muestran los arcos y los pilares planteados en el proyecto original de José Álvarez y su diferenciación en altura con los proyectados por Ignacio Tomás, así como los suplementos de ladrillo y mezcla ideados por éste para reforzar la estructura de la iglesia. En contraposición, el interior de la iglesia (que adquiere una disposición centralizada) aparece concluido siguiendo las medidas propuestas por Tomás (fig. 8), empero edificado hasta el arranque de las bóvedas en piedra, como sugirió Manuel Núñez. Igualmente, las linternas dispuestas por el académico para las naves laterales no se llegaron a realizar debido al reforzamiento de la nave central planteado por Núñez, del mismo modo que las entradas de luz de la misma aparecen cegadas, mermando así el sistema de iluminación planteado por Tomás para la iglesia (fig. 9). Por último, y advirtiendo que el altar mayor diseñado por el académico se encuentra oculto tras un retablo barroco colocado en el siglo XIX, los añadidos de la cúpula y la barroquizante linterna son fruto de las modificaciones dispuestas por el arquitecto sevillano Fernando Rosales a partir de $1816^{87}$ (fig. 10).

La singular apariencia de la parroquia mayor de Santa Cruz de Écija, difícilmente comparable a otros edificios de su época por su fisonomía y por las particularidades de su estado inconcluso, es el resultado del dictamen emitido por diferentes arquitectos a lo largo de su construcción. Aunque la aportación más importante para su edificación y la idea preponderante en su conclusión fue la elaborada por el arquitecto académico Ignacio Tomás, esta obra sintetiza el sentido constructivo de varios autores antagónicos que simbolizan una fase heterogénea de la Arquitectura española. De igual modo, las extraordinarias circunstancias que rodearon su erección ejemplifican la ardua

${ }^{81}$ Écija. Parroquia de Santa Bárbara y San Juan, 25 de agosto de 1792, ARABASF, Comisión de Arquitectura, Informes, Iglesias parroquiales, legajo 2-33-2, exp. 1, s/f.

82 Al Rey. Representacion por D. Ignacio Thomas Arquitecto y Academico de Merito de la Real Academia de San Fernando, 22 de agosto de 1792, ARABASF, Comisión de Arquitectura, Informes, Competencias profesionales, legajo 2-26-1, exp. 2, ff 1r-6r.

83 Junta Particular del 2 de septiembre de 1792, ARABASF, Secretario general, Libros de actas de sesiones particulares, legajo 3-123, ff. 199r-199v.

${ }^{84}$ Écija. Parroquia de Santa Bárbara y San Juan, 3 de septiembre de 1792, ARABASF, Comisión de Arquitectura, Informes, Iglesias parroquiales, legajo 2-33-2, exp. 1, s/f.

85 Hernández Díaz/ Sancho Corbacho/ Collantes de Terán, 1751: 112.

86 Según un proyecto del arquitecto Joaquín Barquín basado en el estudio formal de las obras de José Álvarez. Anteproyecto de terminación de la iglesia de Santa Cruz en Écija, agosto de 1955, Archivo de la Parroquia de Santa Cruz de Écija (APSCE), Planos y Dibujos, exp. 1, s/f.

${ }^{87}$ En concreto, a partir del 19 de julio de 1816. Écija. Santa Cruz, AGAS, Justicia, Pleitos Ordinarios, legajo 10319, exp. 1, ff. 424r y ss. 


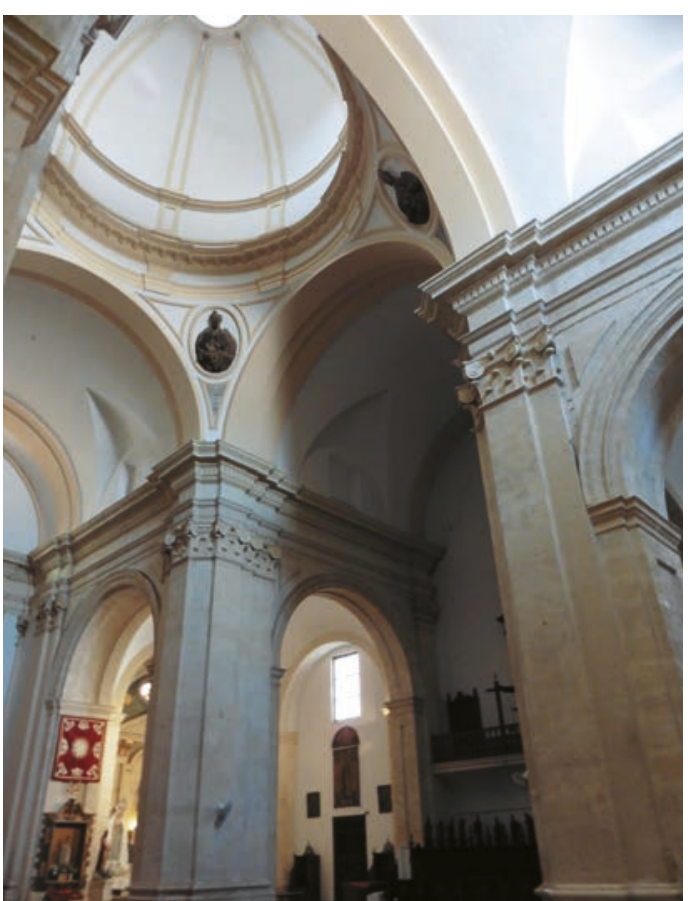

Fig. 9. Interior concluido de la iglesia. Fotografía del autor.

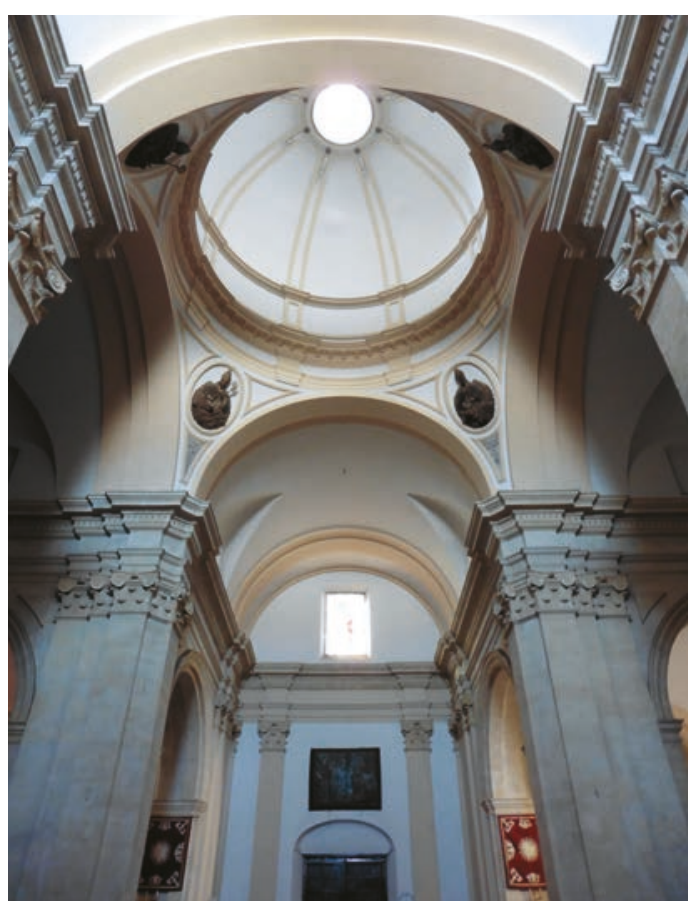

Fig. 10. Transepto y cúpula del interior de la iglesia. Fotografía del autor.

disputa entre los alarifes gremiales y los arquitectos académicos que aconteció en los reinos andaluces en general y en el sevillano en particular a finales del siglo XVIII. Conflicto que marcó el desarrollo de la Arquitectura andaluza de dicho periodo, y cuyas diferentes etapas no sólo quedan plasmadas en la documentación aportada sobre el edificio, sino que excepcionalmente se escenifican formalmente en el mismo. Por todo ello, esta obra adquiere una significación especial como paradigma de un periodo de notables cambios en la Arquitectura española en el que, a pesar de los esfuerzos de la Academia y sus miembros, la gran mayoría de las construcciones sacras andaluzas no pudieron ejecutarse completamente según sus preceptos. La labor de Ignacio Tomás en Écija y su intervención en la iglesia Santa Cruz son paradigma de ello.

\section{BIBLIOGRAFÍA}

Aranda Doncel, Juan (1986): “Un proyecto ilustrado en la Córdoba del siglo XVIII: la escuela de Bellas Artes del obispo Caballero y Góngora”. En: Apotheca, 6, Córdoba, pp. 33-49.

Baena Gallé, Jose Manuel / Hernández Núñez, Juan Carlos (1998): "La Real Academia de San Fernando de Madrid y el Cabildo de la Catedral hispalense: Un proyecto de retablo neoclásico para la Capilla de los Dolores”. En: Laboratorio de Arte, 11, Sevilla, pp. 607-623.

Bails, Benito (1783): Elementos de Matemática. Tomo IX. Parte I. Que trata de la Arquitectura Civil. Madrid: Imprenta de la Viuda de Joaquín Ibarra.

Candau Chacón, María Luisa (1995): "Prólogo". En: Actas del II Congreso de Historia "Écija en el siglo XVIII”. Écija: Ayuntamiento, pp. 3-6.

Falcón Márquez, Teodoro (1979): Pedro de Silva: arquitecto andaluz del siglo XVIII. Sevilla: Diputación.

Fernández González, Alberto (2007): “Trazas y proyectos para el desaparecido retablo mayor de estuco de la iglesia parroquial de Omnium Sanctorum de Sevilla”. En: Laboratorio de Arte, 20, Sevilla, pp. 203-228.

Fernández Martín, María Mercedes (1994): El arte de la madera en Écija en el siglo XVIII. Écija: Ayuntamiento.

Fernández Martín, María Mercedes (1998): "Fiestas en Écija por la proclamación de Carlos IV”. En: Laboratorio de Arte, 11, Sevilla, pp. 591-606.

Fernández Martín, María Mercedes (2000): “Consideraciones sobre la aparición del retablo neoclásico en Écija”. En: Actas del V congreso de historia "Écija en la edad Contemporánea". Écija: Ayuntamiento, pp. 67-74. 
García León, Gerardo (1990): "Planos de Ignacio Tomás para la iglesia de Santa Bárbara de Écija”. En: Laboratorio de Arte, 3, Sevilla, pp. 169-188.

García Melero, José Enrique (1991): "Arquitectura y burocracia: el proceso del proyecto en la Comisión de Arquitectura de la Academia (1786-1808)". En: Espacio, tiempo y forma. Serie VII, Historia del arte, 4, Madrid, pp. 283-348.

García Melero, José Enrique (1995): "Las cárceles españolas de la Ilustración y su censura en la Academia (1777-1808)". En: Espacio, tiempo y forma. Serie VII, Historia del arte, 8, Madrid, pp. 241-272.

García Sepulveda, María Pilar/ Navarrete Martínez, Esteranza (2007): Relación general de académicos (1752-2015). Madrid: Real Academia de Bellas Artes de San Fernando.

Hernández Díaz, José/ Sancho Corbacho, Antonio/ Collantes de Terán, Francisco (1955): Catálogo arqueológico y artístico de la provincia de Sevilla. T. III. Sevilla: La Gavidia.

Higuera Meléndez, José Manuel (2016): "Las intervenciones en Écija del maestro mayor Antonio de Figueroa (17331793): una obligada puesta al día”. En: Isidorianum, 49, Sevilla, pp. 107-158.

Luna Fernández-Aramburu, Rocío/ Serrano Barberá, Concepción (1980): Planos y Dibujos del Archivo de la Catedral de Sevilla (siglos XVI-XX). Sevilla: Diputación.

Morales, Alfredo J. (1996): Hernán Ruiz “el joven”. Madrid: Akal.

Morales, Alfredo J. (2010): La Piel de la Arquitectura. Yeserías sevillanas de los siglos XVII y XVIII. Sevilla: Diputación.

Morales, Alfredo J. (2011): “La Arquitectura en Écija durante los siglos del Barroco”. En: González Gómez, Juan Miguel (coord.): Écija Barroca. Écija: Ayuntamiento, pp. 33-75.

Martín Ojeda, Marina/ García León, Gerardo (1995): La Virgen del Valle. Écija: Sol.

Martín Pradas, Antonio (1995): "Notas para el estudio de la Real Casa de Misericordia de Écija: un proyecto fallido". En: Atrio, 7, Sevilla, pp. 67-75.

Nogales Márquez, Carlos Francisco (2015): El arquitecto Antonio Matías de Figueroa. Tesis doctoral inédita. Sevilla: Universidad.

Ollero Lobato, Francisco (1994): Noticias de Arquitectura. 1761-1780. Sevilla: Guadalquivir.

Ollero Lobato, Francisco (2004): Cultura Artística y Arquitectura en la Sevilla de la Ilustración (1775-1808). Sevilla: Caja San Fernando.

Pérez Ayala, José Manuel (1951): Antonio Caballero y Góngora. Virrey y Arzobispo de Santa Fé. 1723-1796. Bogotá: Imprenta Municipal.

Ponz, Antonio (1792): Viage de España. T. XVII. Madrid: Viuda de D. Joaquín Ibarra.

Quiles García, Fernando (1997): "Datos para una definición de la arquitectura Neoclásica Sevillana". En: Academia, 84, Madrid, pp. 307-333.

Real Academia de San Fernando (1757): Estatutos de la Real Academia de San Fernando. Madrid: Gabriel Ramírez.

Real Academia de San Fernando (1793): Distribución de los premios concedidos por el rey nuestro señor à los discípulos de las tres nobles artes, hecha por la Real Academia de San Fernando en la Junta pública de 20 de agosto de 1793. Madrid: Imprenta de la Viuda de Ibarra.

Real Academia de San Fernando (1796): Distribución de los premios concedidos por el rey nuestro señor á los discípulos de las tres nobles artes, hecha por la Real Academia de San Fernando en la Junta pública de 13 de Julio de 1796. Madrid: Imprenta de la Viuda de Ibarra.

Recio Mir, Álvaro (1998): “Antonio López Aguado y los proyectos neoclásicos para el retablo de la capilla de los Dolores de la Catedral de Sevilla". En: Academia, 86, Madrid, pp. 379-398.

Recio Mir, Álvaro (2009): "El brillante final del Barroco: El retablo rococo". En: Halcón, Fátima/ Herrera, Francisco/ Álvaro, Recio: El retablo sevillano desde sus orígenes a la actualidad. Sevilla: Diputación, Fundación Cajasol y Fundación Real Maestranza de Caballería de Sevilla, pp. 343-388.

Rivas Carmona, Jesús (1986): "Notas para el Neoclásico cordobés". En: Imafronte, 2, Murcia, pp. 25-55.

Ros González, Francisco S. (2000): "El retablo mayor de estuco de la parroquia de Omnium Sanctorum de Sevilla (17911793)". En: Laboratorio de Arte, 13, Sevilla, pp. 153-172.

Ruiz Carrasco, Jesús María (2017): "La iglesia de Santa Bárbara, Ignacio Tomás y la introducción de los preceptos academicistas en la arquitectura sacra astigitana". En: Archivo Hispalense, 303-305, Sevilla, pp. 343-370.

Ruiz Carrasco, Jesús María (2018): "Paradigmas y fundamentos de la conflictiva relación entre la Comisión de Arquitectura y la ciudad de Sevilla (1786-1808)". En: Laboratorio de Arte, 30, Sevilla, pp. 285-302.

Sambricio, Carlos (1985): "Datos sobre los discípulos y seguidores de D. Ventura Rodríguez". En: Estudios sobre Ventura Rodriguez (1717-1785). Madrid: Real Academia de Bellas Artes de San Fernando, pp. 244-304.

Sambricio, Carlos (1986): La Arquitectura española de la Ilustración. Madrid: Consejo Superior de los Colegios de Arquitectos de España.

San Nicolás, Fray Lorenzo de (1736): Arte y uso de Arquitectura. Tercera Impresión. Madrid: Manuel Román.

Sancho Corbacho, Antonio (1952): Arquitectura barroca sevillana del siglo XVIII. Madrid: CSIC.

Serra Masdeu, Anna Isabel (2014): "La biblioteca del arquitecto y académico Ignasi Tomàs Fabregat (c. 1744-1812)". En: Cuadernos de Arte Universidad de Granada, 45, Granada, pp. 129-141.

Valseca Castillo, Ana (1996): De las torres parroquiales de Écija en el siglo XVIII. Sevilla: Diputación.

Fecha de recepción: 01-II-2018

Fecha de aceptación: 06-XI-2018 